\title{
Oportunidades perdidas de avaliação sorológica para a infecção pelo vírus da imunodeficiência humana na rotina de atendimento ambulatorial do Hospital Universitário de Brasília, Distrito Federal
}

\author{
Missed opportunities for serological evaluation of human immunodeficiency virus \\ infection during routine outpatient care at the University Hospital of Brasilia, Federal District
}

\author{
Maria Helena Pereira Pires de Oliveira ${ }^{1}$, Karolinne Cardoso Muniz ${ }^{1}$, \\ Adriano Evangelista Borges ${ }^{1}$, Flávia de Azevedo Belêsa ${ }^{1}$, Eliane Maria Fleury Seidl2, ${ }^{2}$, \\ Wania Maria do Espírito Santo Carvalho ${ }^{3}$ e Gustavo Adolfo Sierra Romero ${ }^{1,3}$
}

\begin{abstract}
RESUMO
Foram estudados os fatores envolvidos na oferta de sorologia para detectar a infecção pelo vírus de imunodeficiência humana, no atendimento de adultos no ambulatório do Hospital Universitário de Brasília, por meio de aplicação de questionários específicos a 53 médicos e 347 usuários. 0 s resultados revelaram que $96,8 \%$ dos usuários identificaram como fator de risco para adquirir a infecção as relações sexuais desprotegidas e 13,6\% desconheciam a possibilidade de transmissão vertical. Em relação à exposição dos usuários aos fatores de risco, $88,2 \%$ praticaram relações sexuais desprotegidas, 22,2\% tiveram diagnóstico de outras doenças de transmissão sexual e 22,2\% tinham recebido transfusões sangüíneas. Os fatores de risco mais questionados pelos médicos foram a prática de relações sexuais desprotegidas e o diagnóstico prévio de hepatite B ou C (35,9\% para ambos). Dezoito por cento dos usuários receberam oferta de testes no Hospital Universitário de Brasília; 15,8\% foram testados e 7,4\% dos indivíduos testados não tiveram acesso ao resultado. Noventa e um por cento dos médicos referiram sentir-se confortáveis ao oferecer testes e apenas 30,4\% oferecem-nos rotineiramente. 0 estudo confirma a perda de oportunidades de testagem sorológica para detectar a infecção no Hospital Universitário de Brasília e reforça a necessidade de implementar medidas para corrigir o problema.
\end{abstract}

Palavras-chaves: Vírus da imunodeficiência humana. Síndrome da imunodeficiência humana. Sorologia. Prevenção. Oportunidades perdidas.

\begin{abstract}
The factors involved in the provision of serological testing to detect human immunodeficiency virus infection, for adults within the outpatient care setting at the University Hospital of Brasilia, were studied. Specific questionnaires were applied to 53 physicians and 347 users. The results showed that $96.8 \%$ of the users identified unprotected sexual intercourse as a risk factor for acquiring this infection and that $13.6 \%$ were unaware of possibility of vertical transmission. Regarding users' exposure to risk factors, $88.2 \%$ practiced unprotected sexual intercourse, $22.2 \%$ had a diagnosis of other sexually transmitted diseases and $22.2 \%$ had received blood transfusions. The risk factors that physicians asked about most frequently were unprotected sexual practices and previous diagnoses of hepatitis B or C (35.9\% for each). Eighteen percent of the users had been offered tests at the University Hospital of Brasilia; $15.8 \%$ underwent tests and 7.4\% of the individuals tested had not had access to the results. Ninety-one percent of the physicians said that they felt comfortable about offering tests, while only $30.4 \%$ offered them routinely. The study confirms that opportunities for serological testing to detect this infection at the University Hospital of Brasilia have been missed and reinforces the need to implement measures to correct this problem.
\end{abstract}

Key-words: Human immunodeficiency virus. Acquired immunodeficiency syndrome. Serology. Prevention. Missed opportunities.

\footnotetext{
1. Núcleo de Medicina Tropical, Faculdade de Medicina, Universidade de Brasília, Brasília, DF. 2. Instituto de Psicologia, Universidade de Brasília, Brasília, DF. 3. Hospital Universitário de Brasília, Brasília, DF.

Apoio financeiro: Programa de Iniciação Científica do CNPq, Brasil.

Endereço para correspondência: Prof. Gustavo Adolfo Sierra Romero. Núcleo de Medicina Tropical/UnB. Campus Universitário Darcy Ribeiro, Asa Norte, Caixa Postal 04517, 70904-970, Brasília, DF.

Tel: $55613273-5008$

e-mail: gromero@unb.br

Recebido para publicação em 23/10/2008

Aceito em 05/03/2009
}

A infecção pelo vírus da imunodeficiência humana (HIV) constitui uma pandemia em expansão. Segundo a Organização Mundial de Saúde, até dezembro de 2007, havia 33 milhões de pessoas infectadas pelo HIV em todo o mundo, das quais, 1,6 milhões viviam na América Latina e 620.000 no Brasil ${ }^{412}$.

Entre 12 e $58 \%$ das pessoas detectadas com a infecção pelo HIV, nos Estados Unidos da América do Norte, desenvolvem a síndrome de imunodeficiência adquirida (Aids) até um ano após o diagnóstico, com variações dependendo da faixa etária 
e o tipo de exposição ao HIV ${ }^{1}$, indicando que permaneceram assintomáticos por um longo período sem que a infecção fosse detectada. Existem evidências de que níveis elevados da carga viral estão diretamente relacionados ao risco de transmissão do HIV. Níveis mais elevados de viremia são encontrados na infecção aguda, em estágios avançados da doença e durante a co-infecção com outras doenças tais como a tuberculose ${ }^{7811}$. Tal fato é de suma importância, visto que, além de ser mais difícil o manejo dos pacientes em estágios avançados da doença, essas pessoas podem ser mais relevantes como transmissores da infecção.

Nessa perspectiva, o Programa Brasileiro de Doenças Sexualmente Transmissíveis e Aids (DST/Aids) inclui entre as suas estratégias o incentivo à realização de exames sorológicos para diagnosticar a infecção, garantidas as ações de aconselhamento pré e pós-teste. No final da década de 80 , os centros de testagem e aconselhamento (CTA) surgiram para oferecer exames gratuitos, participação voluntária e sigilo. Em 2003, a campanha Fique Sabendo! foi lançada com o objetivo de aumentar em 150\% o número de indivíduos testados no Brasil em um período de 3 anos 9 .

As estratégias de estímulo à avaliação sorológica obtiveram algum sucesso. 0 percentual de pessoas testadas no Brasil cresceu de 20\% em 1998 para 32,9\% em 2005. No entanto, a maior parte da população avaliada foi de mulheres entre 25 e 39 anos, situação explicada pela incorporação do exame anti-HIV em serviços de pré-natal. Esses dados sugerem que homens e mulheres nãogestantes ainda não foram alcançados significativamente pelos esforços governamentais 9 .

Ademais, o aconselhamento pré e pós-teste surgiu como uma estratégia de prevenção, trabalhando a avaliação de riscos e a reflexão sobre medidas preventivas viáveis para cada pessoa que pretende realizar a testagem. 0 aconselhamento é visto atualmente como um momento de educação em saúde e tem sido eficaz na redução de doenças sexualmente transmissíveis ${ }^{6}$.

0 estudo teve como objetivos: descrever o conhecimento de usuários de um hospital de ensino sobre a transmissão do HIV e a freqüiência de fatores de risco para adquirir a infecção; assim como, determinar a frequiência de oferta e de aceitação da avaliação sorológica; a frequiência com que os médicos questionam os pacientes sobre fatores de risco para adquirir a infecção; e os fatores que levam o profissional a indicar a avaliação sorológica.

\section{PACIENTES E MÉTODOS}

O estudo foi realizado no Hospital Universitário de Brasília (HUB) onde são atendidas rotineiramente entre 280 e 300 pessoas com HIV/Aids. 0 estudo foi realizado no centro de atendimento ambulatorial de adultos e foi dividido em duas etapas. A primeira etapa, por meio de questionário aplicado por entrevistador treinado, no período de junho de 2006 a janeiro de 2007, após a realização da consulta, abordou usuários de todas as clínicas de especialidades, exceto a de doenças infecciosas e parasitárias. A segunda etapa foi realizada por meio de questionários auto- aplicáveis respondidos por médicos selecionados aleatoriamente no mesmo local. As 23 especialidades médicas foram agrupadas em especialidades clínicas, cirúrgicas ou gineco-obstétricas, para facilitar a análise.

0 tamanho da amostra de usuários foi estimado para detectar eventos que se apresentam com frequiência de pelo menos 5\%, considerando inclusive os potenciais efeitos de conglomerados durante a amostragem.

O questionário destinado aos usuários abordou: a especialidade onde o entrevistado consulta e o número de consultas já realizadas; características sócio-demográficas; conhecimentos sobre a transmissão do HIV; exposição a fatores de risco para a transmissão do HIV; fatores de risco questionados pelos médicos durante as consultas; realização prévia da sorologia para HIV e acesso ao resultado; e realização de testes anti-HIV durante o acompanhamento pré-natal. Além disso, foi oferecida ao entrevistado a possibilidade de receber aconselhamento e realizar os testes sorológicos no HUB. Ao final da entrevista, o usuário foi convidado a fazer comentários, sugestões e tirar dúvidas, criando um momento de educação em saúde.

0 instrumento respondido pelos médicos abordou: as características gerais quanto ao sexo, idade, especialidade e vínculo institucional; fatores de risco interrogados durante as consultas; e aspectos valorizados para a oferta de testagem sorológica anti-HIV.

0 banco de dados e a análise foi realizada utilizando-se 0 software SPSS® versão 11.0. 0 estudo foi aprovado pelo Comitê de Ética em Pesquisa da Faculdade de Medicina da Universidade de Brasília (Processo 81/2005 aprovado em 07/12/2005) e cumpriu as recomendações da Resolução 196/96 do Conselho Nacional de Saúde que regulamenta a pesquisa com seres humanos no Brasil. Todos os pacientes e médicos envolvidos na pesquisa participaram voluntariamente e assinaram o termo de consentimento livre e esclarecido.

\section{RESULTADOS}

Avaliação com os usuários. Dos 347 entrevistados, atendidos em 23 especialidades médicas, 119 (34\%) eram do sexo masculino e 229 (66\%) eram do sexo feminino. A mediana de idade dos participantes foi de 46,4 anos, com variação de 18 a 80 anos. 0 perfil dos usuários mostrou que 40,3\% tinham ensino fundamental incompleto e 9,3\% ingressaram em curso superior. Quanto à situação conjugal, 62,2\% estavam casados ou em união consensual, 21,3\% eram solteiros, 9,5\% divorciados e 6,9\% viúvos. Em relação ao emprego, unicamente $28,2 \%$ trabalhavam com vínculo fixo com os direitos trabalhistas garantidos.

Quarenta por cento dos entrevistados já haviam consultado de duas a cinco vezes, na mesma especialidade, 37,5\% acima de 10 vezes, $12,2 \%$ entre seis e 10 vezes e $10,5 \%$ tinham realizado a sua primeira consulta naquele momento, antes da entrevista.

A avaliação do conhecimento dos 347 usuários sobre os fatores de risco para a transmissão do HIV está apresentada na Tabela 1. 
TABELA 1

Conhecimento de adultos atendidos no Hospital Universitário de Brasília sobre fatores de risco para transmissão do vírus da imunodeficiência humana. Brasília. 2006-2007.

\begin{tabular}{|c|c|c|c|c|c|c|}
\hline \multirow[b]{3}{*}{ Aspecto avaliado } & \multicolumn{6}{|c|}{ Resposta* } \\
\hline & \multicolumn{2}{|c|}{$\operatorname{sim}$} & \multicolumn{2}{|c|}{ não } & \multicolumn{2}{|c|}{ não sabe } \\
\hline & $\mathrm{n}^{\mathrm{o}}$ & $\%$ & $\mathrm{n}^{0}$ & $\%$ & $\mathrm{n}^{\mathrm{o}}$ & $\%$ \\
\hline Picada de inseto & 87 & 25,1 & 247 & 62,5 & 43 & 12,4 \\
\hline Compartilhamento de seringas & 333 & 96,0 & 8 & 2,3 & 6 & 1,7 \\
\hline Uso de banheiros públicos & 80 & 23,0 & 239 & 68,9 & 28 & 8,1 \\
\hline Compartilhamento de talheres, copos ou refeições & 58 & 16,7 & 266 & 76,7 & 23 & 6,6 \\
\hline Não usar preservativos nas relações sexuais & 336 & 96,8 & 8 & 2,3 & 3 & 0,9 \\
\hline Da mãe para o filho durante a gravidez, parto ou amamentação & 300 & 86,5 & 21 & 6,0 & 26 & 7,5 \\
\hline
\end{tabular}

*total de 347 adultos entrevistados.

Quanto à exposição a fatores de risco relatados pelos usuários, 88,2\% tiveram relações sexuais sem uso de preservativo, $22,2 \%$ tinham recebido transfusões sangüíneas, $22,2 \%$ tiveram diagnóstico prévio de doenças sexualmente transmissíveis, $2,3 \%$ compartilhavam seringas e 1,2\% fizeram transplante de órgãos.

Os resultados das respostas de 328 usuários que efetivamente realizaram a parte da entrevista sobre se, durante qualquer consulta prévia na especialidade, o médico havia perguntado sobre os fatores de risco para a transmissão do HIV, estão apresentados na Tabela 2. As respostas observadas foram semelhantes entre os grupos de especialidades, exceto porque os pacientes atendidos nas especialidades gineco-obstétricas foram mais questionados em relação aos antecedentes de doenças sexualmente transmissíveis e receberam maior oferta de testagem para HIV.

As respostas ao questionamento sobre a realização do teste anti-HIV revelaram que, 28,2\% foram testados fora do HUB (CTA 0,6\%, hemocentro 4,3\%, hospitais públicos 8,4\%, centros de saúde 7,2\%, hospitais/clínicas ou laboratórios particulares 6,1\%), e 5,1\% não tiveram acesso ao resultado. No HUB, 17,6\% dos usuários receberam oferta, 15,8\% foram testados e 7,4\% não tiveram acesso ao resultado.

Vinte e nove usuárias referiram ter estado grávidas nos últimos cinco anos. Durante o pré-natal, o teste foi oferecido para 79,3\% delas, das quais $65,5 \%$ realizaram o teste e $100 \%$ tiveram acesso ao resultado.

Ao final da entrevista, 52,4\% dos usuários revelaram que gostariam de ser esclarecidos sobre o assunto. Após o aconselhamento, 41,2\% aceitaram a realização do teste.

Avaliação com os médicos. Dos 53 participantes, 31 eram do sexo feminino e 22 do sexo masculino. A mediana de idade dos médicos foi 32,9 anos, idades variando de 24 a 62 anos com maior freqüência de profissionais de 24 a 32 anos (38 profissionais). Quanto ao vínculo com a instituição, 30 eram residentes e 23 estafes.

TABELA 2

Frequiência com que os médicos perguntam aos pacientes durante a consulta ambulatorial sobre fatores de risco para adquirir o vírus de imunodeficiência humana segundo a especialidade avaliada. Hospital Universitário de Brasília. Brasília. 2006-2007.

\begin{tabular}{|c|c|c|c|c|c|c|c|c|}
\hline \multirow[t]{3}{*}{ Aspecto avaliado } & \multicolumn{8}{|c|}{ Especialidades } \\
\hline & \multicolumn{2}{|c|}{ clínicas* } & \multicolumn{2}{|c|}{ cirúrgicas** $^{* *}$} & \multicolumn{2}{|c|}{ gineco-obstétricas $^{* * *}$} & \multicolumn{2}{|c|}{ total } \\
\hline & $\mathrm{n}^{0}$ & $\%$ & $\mathrm{n}^{0}$ & $\%$ & $\mathrm{n}^{0}$ & $\%$ & $\mathrm{n}^{0}$ & $\%$ \\
\hline Transfusão de hemoderivados & 36 & 19,6 & 30 & 26,6 & 9 & 29,0 & 75 & 22,9 \\
\hline Transplante de órgãos & 31 & 16,9 & 20 & 17,7 & 6 & 19,4 & 57 & 17,4 \\
\hline Diagnóstico de Hepatite B ou C & 66 & 35,9 & 42 & 37,2 & 11 & 35,5 & 119 & 36,3 \\
\hline Diagnóstico de DST & 43 & 23,4 & 26 & 23,0 & 16 & 51,6 & 85 & 25,9 \\
\hline Compartilhamento de seringas & 25 & 13,6 & 21 & 18,6 & 6 & 19,4 & 52 & 15,9 \\
\hline Relações sexuais desprotegidas & 25 & 35,9 & 21 & 37,2 & 11 & 35,5 & 57 & 36,3 \\
\hline Realização do teste anti-HIV & 27 & 14,7 & 18 & 16 & 9 & 29,0 & 54 & 16,5 \\
\hline Total & 184 & 56,1 & 113 & 34,4 & 31 & 9,5 & 328 & 100 \\
\hline
\end{tabular}

* Especialidades clínicas: dermatologia, gastroenterologia, pneumologia, cardiologia, nefrologia, endocrinologia, oncologia, clínica médica, alergologia, geriatria, reumatologia, neurologia, psiquiatria. Percentagens calculadas sobre o total de 184 entrevistados.

** Especialidades cirúrgicas: neurocirurgia, cirurgia vascular, urologia, cirurgia geral, proctologia, otorrinolaringologia, ortopedia. Percentagens calculadas sobre o total de 113 entrevistados.

***Especialidades gineco-obstétricas: ginecologia, obstetrícia, mastologia. Percentagens calculadas sobre o total de 31 entrevistados

DST: doença sexualmente transmissível, HIV: vírus da imunodeficiência humana. 
A frequiência com a qual os 53 médicos referiram questionar os pacientes sobre fatores de risco para adquirir a infecção pelo HIV está demonstrada na Figura 1.
Quando interrogados sobre a política governamental de testagem para HIV, 30,2\% dos médicos demonstraram desconhecê-la. Os dados relacionados à investigação sobre

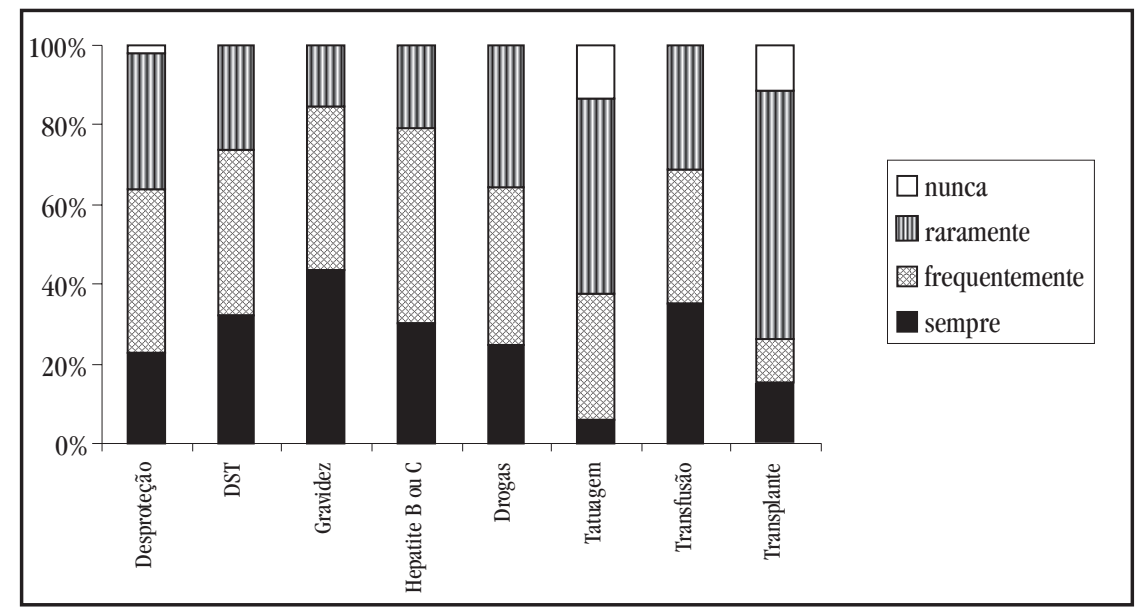

FIGURA 1

Frequiência com que os médicos questionam os pacientes sobre fatores de risco para a infecção pelo vírus da imunodeficiência humana no ambulatório do Hospital Universitário de Brasília. Brasília. 2006-2007.

fatores de risco e à solicitação de exames sorológicos para HIV estão demonstrados na Tabela 3. Médicos residentes e estafes apresentaram comportamento semelhante nestes aspectos.

TABELA 3

Frequiência dos aspectos relacionados com a atitude dos médicos na solicitação do teste sorológico para a infecção pelo vírus de imunodeficiência humana no ambulatório do Hospital Universitário de Brasília. Brasília. 2006-2007.

\begin{tabular}{|c|c|c|c|c|c|c|}
\hline \multirow[b]{2}{*}{ Aspecto avaliado } & \multicolumn{2}{|c|}{$\begin{array}{c}\text { Respostas positivas dos } \\
\text { estafes } n^{0}=23\end{array}$} & \multicolumn{2}{|c|}{$\begin{array}{l}\text { Respostas positivas dos } \\
\text { residentes } \mathrm{n}^{0}=30\end{array}$} & \multicolumn{2}{|c|}{$\begin{array}{c}\text { Total de respostas } \\
\text { positivas } \mathrm{n}^{0}=53\end{array}$} \\
\hline & $\mathrm{n}^{0}$ & $\%$ & $\mathrm{n}^{0}$ & $\%$ & $\mathrm{n}^{0}$ & $\%$ \\
\hline Precisa suspeitar que o paciente está infectado para oferecer o teste anti-HIV? & 11 & 48,0 & 17 & 56,7 & 28 & 53,8 \\
\hline Sente-se confortável questionando o paciente sobre fatores de risco para adquirir HIV? & 22 & 95,6 & 26 & 86,7 & 48 & 90,6 \\
\hline Sente-se confortável oferecendo aos pacientes o teste anti-HIV? & 21 & 91,3 & 24 & 80,0 & 45 & 84,9 \\
\hline Entrevista o paciente sobre fatores de risco para adquirir HIV durante a primeira consulta? & 8 & 34,8 & 14 & 46,7 & 22 & 41,5 \\
\hline Oferece rotineiramente o teste aos pacientes? & 7 & 30,4 & 11 & 36,7 & 18 & 34,0 \\
\hline
\end{tabular}

HIV: vírus da imunodeficiência humana.

\section{DISCUSSÃo}

As amostras de usuários e médicos foram de conveniência e os participantes foram abordados de acordo com o tempo disponibilizado pelos entrevistadores para o estudo. Portanto, existe a possibilidade de viés de seleção pela exclusão daqueles usuários que consultaram e dos médicos que atenderam exclusivamente nos períodos nos quais os entrevistadores não estavam disponíveis. Por outro lado, a entrevista foi oferecida a todos os usuários e médicos sem aplicação de critérios de exclusão, exceto porque não foram avaliados os médicos e pacientes dos ambulatórios de doenças infecciosas e parasitárias com 0 intuito de evitar 0 viés que reduzisse a estimativa de falta de oferta dos exames, considerando que a maioria da clientela atendida no serviço de doenças infecciosas é de pessoas vivendo com HIV/Aids.
0 instrumento utilizado para entrevista dos usuários foi desenvolvido pelos pesquisadores e testado previamente em usuários atendidos pela Clínica de Doenças Infecciosas e Parasitárias do HUB, que não seriam incluídos no estudo, preparando a equipe para a entrevista e com o objetivo de aprimorar a capacidade dos entrevistadores para lidar com assuntos relacionados à infecção pelo HIV. $O$ instrumento foi construído com perguntas simples adequadas à população entrevistada, tendo em vista que se esperava que uma proporção importante dos usuários tivesse nível educacional baixo, o que foi constatado com 40,3\% deles apresentando ensino fundamental incompleto.

Em relação à potencialidade de induzir algum tipo de respostas pelos entrevistadores, o modelo da pesquisa inevitavelmente incorreu nesse risco. Apesar disso, considera-se que seria plausível que os entrevistados tentassem responder no sentido de atender às expectativas dos profissionais de saúde relatando mais freqüentemente o uso de práticas mais seguras ou negando 
a presença de comportamentos de risco para alcançar maior aceitabilidade social. Este fenômeno, que não é mensurável, poderia ter tido um impacto na subestimação da frequiência de práticas com risco elevado para adquirir HIV ou de práticas de oferta de exames para HIV em níveis aceitáveis para um centro de referência como o HUB. No entanto, as entrevistas foram capazes de detectar problemas de comportamentos de risco nos usuários e de situações negativas no processo de oferta dos exames pelos médicos, que embora possam estar subestimados, se forem considerados como reais nas magnitudes detectadas, revelam claramente a gravidade do problema. 0 questionário destinado aos médicos foi auto-aplicado, portanto, foi menos vulnerável aos problemas de indução de respostas pelos entrevistadores, mas, certamente sofreu o impacto da explicitação do objeto da pesquisa no termo de consentimento livre e esclarecido.

Apesar das limitações, o estudo evidenciou aspectos importantes que merecem análise. Sobre o conhecimento das situações de risco para a transmissão do HIV, boa parte dos usuários tem consciência do risco envolvido no compartilhamento de seringas e na relação sexual desprotegida. Fato que poderia ser explicado pelo maior destaque dado a essas questões nas campanhas, como por exemplo, a Carnaval 2007: Prazer com tranqüilidade $^{4}$. Ao mesmo tempo, nota-se uma percepção equivocada sobre o risco de transmissão em banheiros públicos e o compartilhamento de talheres e refeições, podendo gerar preconceito contra a população vivendo com HIV/Aids.

É evidente, pelos resultados do estudo, que não existem no HUB práticas consolidadas ou roteiros para a realização de uma anamnese voltada para a investigação de situações de risco de infecção pelo HIV. Apesar de manifestarem que se sentiam confortáveis quanto ao processo de oferta de testagem, os médicos não questionaram os pacientes sobre esse tema e deixaram de oferecer o teste sorológico. 0 comportamento semelhante entre os médicos residentes e os estafes mostra que no HUB talvez seja reproduzido um modelo onde, independentemente da experiência no atendimento, exista uma cultura de baixa oferta de testes anti-HIV, o que se torna ainda mais preocupante já que as novas gerações de especialistas apresentam atitude semelhante à dos seus tutores. Esse fenômeno mostra a necessidade de discutir com esses profissionais, no sentido de identificar e reduzir possíveis preconceitos e as causas que os sustentam. 0 uso de metodologia qualitativa para conhecer as causas da falta de oferta de testagem pelos profissionais médicos poderá ajudar ao desenvolvimento de estratégias para superar ou minimizar o problema ${ }^{610}$.

Em estudo realizado nos Estados Unidos da América do Norte para avaliar a perda de oportunidades para o diagnóstico do HIV em uma população de pessoas vivendo com HIV/Aids, durante contatos prévios com o sistema de atendimento em saúde, detectou-se que mesmo quando o paciente apresenta indícios de infecção pelo HIV, os médicos nem sempre consideram a necessidade de investigação sorológica ${ }^{3}$.

A implementação efetiva da política governamental de oferta ampla de testagem para HIV é essencial para reduzir o impacto negativo da perda de oportunidades de testagem durante o contato das pessoas com o sistema de saúde. Vários estudos têm demonstrado que existe um espaço a ser abordado nesse sentido ${ }^{23}$. No segmento de mulheres gestantes, para as quais, a meta de cobertura de testagem o mais precocemente possível é de $100 \%$, a perda de oportunidades é de particular significado. No presente estudo, das mulheres que informaram gestações no período de cinco anos antes da entrevista, apenas 79,3\% relataram a oferta durante o pré-natal; $\mathrm{e}$ apesar de que o número de entrevistadas foi baixo, a observação de que aproximadamente uma de cada quatro gestantes não foi testada apropriadamente, reflete a gravidade da situação. 0 estudo mostra a necessidade de trabalhar a questão nos vários cenários de prática do Sistema Único de Saúde, reforçando a oferta ampla de testagem acompanhada de práticas baseadas na detecção e aconselhamento sobre as situações de risco. A incorporação de questões dirigidas para detectar tais situações, durante a anamnese rotineira, deve ser considerada essencial para o sucesso da estratégia.

\section{AGRADECIMENTOS}

Ao Programa de Iniciação Científica do Conselho Nacional de Desenvolvimento Científico e Tecnológico - CNPq.

\section{REFERÊNCIAS}

1. Centers for Disease Control and Prevention. HIV/AIDS Surveillance Report. Atlanta: U.S. Department of Health and Human Services, Centers for Disease Control and Prevention. Vol. 18, 2006. Disponível em: http://www.cdc.gov/hiv/ topics/surveillance/resources/reports/2006report/pdf/2006SurveillanceReport. pdf [acesso em 04/10/2008]

2. Jenkins TC, Gardner EM, Thrun MW, Cohn DL, Burman WJ. Risk-based Human Immunodeficiency Virus (HIV) testing fails to detect the majority of HIV-infected persons in medical care settings. Sexually Transmitted Diseases 33: 329-333, 2006

3. Liddicoat RV, Horton NJ, Urban R, Maier E, Christiansen D, Samet JH. Assessing missed opportunities for HIV testing in medical settings. Journal of General Internal Medicine 19:349-56, 2004.

4. Ministério da Saúde. Aids no mundo. Programa Nacional de DST e Aids, 2007. Disponível em: http://www.aids.gov.br/data/Pages/LUMIS63943F78PTBRIE.htm [consultado em 11/09/2008].

5. Ministério da Saúde. Carnaval 2007: prazer com tranqüilidade. Programa Nacional de DST e Aids, 2007. Disponível em: http://www.aids.gov.br/data/Pages/00B107 0ITEMID1B7DC3DD216A4460A1EB759E6EF2584FPTBRIE.htm [consultado em 11/09/2008].

6. Miranda KCL, Barroso MGT. HIV/AIDS counseling: analysis based on Paulo Freire. Revista Latino Americana de Enfermagem 15:100-105, 2007.

7. Miró JM, Sued O, Plana M, Pumarola T, Gallart T. Avances en el diagnóstico y tratamiento de la infección aguda por el VIH-1. Enfermedades Infecciosas y Microbiología Clínica 22: 643-659, 2004.

8. Orenstein R. Presenting Syndromes of Human Immunodeficiency Virus. Mayo Clinic Proceedings 77: 1097-1102, 2002

9. Paiva V, Pupo LR, Barbosa R. The right to prevention and the challenges of reducing vulnerability to HIV in Brazil. Revista de Saúde Pública 40 (supl):109-119, 2006.

10. Simon V, Ho DD, Abdool Karim Q. HIV/AIDS epidemiology, pathogenesis, prevention and treatment. Lancet 368: 489-504, 2006.

11. Serapioni M. Métodos qualitativos e quantitativos na pesquisa social em saúde: algumas estratégias para a integração. Ciência e Saúde Coletiva 5:187-192, 2000.

12. World Health Organization. AIDS epidemic update, 2007. http://data.unaids.org/ pub/EPISlides/2007/2007_epiupdate_en.pdf [acessado em 14/07/2008]Y. 